\title{
Analysis of the Robin-Dirichlet iterative procedure for solving the Cauchy problem for elliptic equations with extension to unbounded domains
}

\author{
Pauline Achieng
}





\title{
Analysis of the Robin-Dirichlet iterative procedure for solving the Cauchy problem for elliptic equations with extension to unbounded domains
}

\author{
Pauline Achieng
}

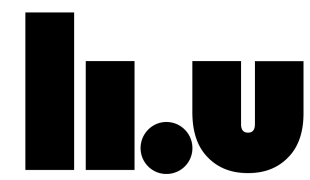


Linköping Studies in Science and Technology. Licenciate Thesis No. 1891

Analysis of the Robin-Dirichlet iterative procedure for solving the Cauchy problem for elliptic equations with extension to unbounded domains

Copyright (C) Pauline Achieng, 2020

Department of Mathematics

Linköping University

SE-581 83 Linöping, Sweden

Email: pauline.achieng@liu.se

ISSN $0280-7971$

ISBN 978-91-7929-756-5

Printed by LiU-Tryck, Linköping, Sweden, 2020 


\section{Abstract}

In this thesis we study the Cauchy problem for elliptic equations. It arises in many areas of application in science and engineering as a problem of reconstruction of solutions to elliptic equations in a domain from boundary measurements taken on a part of the boundary of this domain. The Cauchy problem for elliptic equations is known to be ill-posed.

We use an iterative regularization method based on alternatively solving a sequence of well-posed mixed boundary value problems for the same elliptic equation. This method, based on iterations between Dirichlet-Neumann and Neumann-Dirichlet mixed boundary value problems was first proposed by Kozlov and Maz'ya [13] for Laplace equation and Lame' system but not Helmholtz-type equations. As a result different modifications of this original regularization method have been proposed in literature. We consider the Robin-Dirichlet iterative method proposed by Mpinganzima et.al [3] for the Cauchy problem for the Helmholtz equation in bounded domains.

We demonstrate that the Robin-Dirichlet iterative procedure is convergent for second order elliptic equations with variable coefficients provided the parameter in the Robin condition is appropriately chosen.

We further investigate the convergence of the Robin-Dirichlet iterative procedure for the Cauchy problem for the Helmholtz equation in a an unbounded domain. We derive and analyse the necessary conditions needed for the convergence of the procedure.

In the numerical experiments, the precise behaviour of the procedure for different values of $k^{2}$ in the Helmholtz equation is investigated and the results show that the speed of convergence depends on the choice of the Robin parameters, $\mu_{0}$ and $\mu_{1}$. In the unbounded domain case, the numerical experiments demonstrate that the procedure is convergent provided that the domain is truncated appropriately and the Robin parameters, $\mu_{0}$ and $\mu_{1}$ are also chosen appropriately. 
To my family,

for their continual support and love. 


\section{Acknowledgments}

I would like to thank my supervisors, Vladimir Kozlov and Fredrik Berntsson for their support, guidance, patience, motivation and collaboration during my studies at Linköping University. I am truly grateful for all the time we spent discussing and challenging me to become a strong mathematician.

I also extend my sincere gratitude to my fellow graduate students and the staff at the Department of Mathematics for the support and the conducive environment for studies.

I would like to thank my mentor and advisor, Charles Nyandwi, whom I have worked with at the University of Nairobi since first year undergraduate. Thank you for mentoring me, believing in me and ensuring that I remained focused on this dream.

To my family, Mom, Dad, brother and sister, you have always been my rock and my emotional support system. Thank you for always encouraging and cheering me up especially during the low moments when I needed you most.

This work has been financed by the International Science Programme (ISP) and the Eastern Africa Universities Mathematics Programme (EAUMP). Special thanks to the coordinators; Leif Abrahamson, Patrick Weke, Jared Ongaro and other ISP staff for always ensuring that my stay was comfortable during my visits to Sweden. 


\section{Contents}

Abstract ...................... i

Acknowledgments . . . . . . . . . . . . . . . iii

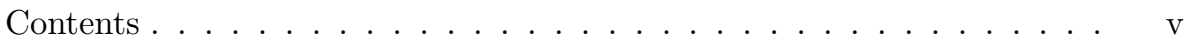

1 Introduction 1

1.1 Ill-posed problems . . . . . . . . . . . . . . . . . . 1

1.1.1 Cauchy problems for elliptic equations . . . . . . . 2

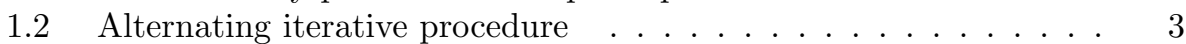

2 Summary of Papers $\quad 7$

References ...................... 9

$\begin{array}{ll}\text { PAPER I } & 11\end{array}$

$\begin{array}{ll}\text { PAPER II } & 33\end{array}$ 


\section{1 - Introduction}

Inverse and ill-posed problems have been widely and extensively studied since early 20th century. Hadamard in [10], was the first to define the notion of a wellposed problem for a differential equation. He further demonstrated this notion using the Cauchy problem for the Laplace equation. He however believed that ill-posed problems did not have physical applications.

The need to study ill-posed problems quickly grew over the years because of the increase in the number of ill-posed problems in science and industry. There was need to theoretically understand and find solutions to these problems. Significant and tremendious growth took place in the 1960s. Tikhonov, is among the many scientists in literature known for their substantial contributions in the progress and growth of the theory of ill-posed problems. In [20, 19], he proposed stable methods for solving these incorrectly formulated problems.

Due to the invention of powerful computers in the 1960s, many researchers, especially mathematicians, turned their attention to the study of inverse problems. Many problems in classical mathematics are ill-posed and prior to the invention of powerful computers it was difficult to solve them. Therefore, driven by the inventions, the need for solutions and theoretical understanding, much studies and different techniques for tackling inverse problems have been developed in literature. See $[17,4,15,18]$ for more details on inverse and ill-posed problems and the different regularization techniques developed for solving these problems.

Consequently, inverse problems arising in other fields of science like medicine, physics, geology etc. can be formulated mathematically as Cauchy problems for partial differential equations. For example in medicine, application arises in Computerized Tomography (CT) where density is recovered from X-ray measurements taken from a cross-section of the human body [8]. In physics applications arise in acoustics and electromagnetic waves i.e detection of source of acoustical noise inside the cabin of a midsize aircraft from measurements of acoustical pressure field inside the cabin $[6,7]$. More information on applications in inverse acoustics and electromagnetic scattering can be found in $[5,12]$.

Let us recall Hadamard's definition of well-posed problems.

\subsection{III-posed problems}

Following Hadamard's definition [9], a well-posed problem must satisfy the following properties:

1. The problem must have a solution (existence)

2. The solution of the problem must be unique (uniqueness), and

3. The problem must depend continuously on the given data (stability) 
In order for this definition to be mathematically precise, the function space for the solution and the notion of continuity must be well defined. In the event that any of these three conditions is violated, then the problem is said to be ill-posed.

Violation of the first condition implies that the problem does not have a solution within the desired space. This problem can be fixed. In the case of exact Cauchy data, existence of the solution can be imposed by redefining the notion of a solution. For example, if one cannot find a classical solution to a problem modelled by a partial differential equation, then one can seek for a solution in a weak sense.

Violation of the second condition implies that a problem has more than one solution in the space and the challenge is to choose the appropriate one. However this can be fixed by implementing a priori information about the solution or in the case of incomplete model, adding additional information to the model.

Violation of the third condition implies that the solution procedure is unstable. Unstable means that small perturbations of the Cauchy data can lead to huge deviation of the numerical solution from the exact solution. This condition is the most difficult to deal with because measurement errors and model errors are impossible to avoid. Therefore problems that violate this condition cannot be solved using classical numerical methods.

Cauchy problems for elliptic equations are known to be ill-posed. In the next section, we give a brief description of these problems, give an example and analyse the regularization methods used to approximate their solutions.

\subsubsection{Cauchy problems for elliptic equations}

An elliptic Cauchy problem is a boundary value problem that constitutes a second order linear partial differential equation that satisfy certain conditions given on the boundary of the domain (bounded or unbounded). Let us consider the general Cauchy problem for an elliptic equation in a domain $\Omega$.

Let $\Omega$ be a bounded domain in $R^{n}$ with a Lipschitz boundary $\Gamma$ and $\Gamma_{0}$ be an open part of the boundary $\Gamma$. The Cauchy problem is to find a solution $u$ which satisfies:

$$
\begin{cases}L u=0 & \text { in } \quad \Omega, \\ u=f & \text { on } \quad \Gamma_{0}, \\ \partial_{\nu} u=g & \text { on } \quad \Gamma_{0},\end{cases}
$$

where $L$ is a second order elliptic operator in $\Omega, \nu$ is the outward unit normal to $\Gamma_{0}$ and $\partial_{\nu}$ is the normal derivative of $u$ on $\Gamma_{0} . f$ and $g$ are the Dirichlet and Neumann data on $\Gamma_{0}$.

For elliptic equations defined on unbounded (infinite) domains, conditions at infinity are usually imposed. For example a-priori bound for the solution or requiring that the solution decay exponentially to zero.

The Laplace operator: $L u=\Delta u$, the Helmholtz operator: $L u=\left(\Delta+k^{2}\right) u$ and the modified Helmholtz operator: $L u=\left(\Delta-k^{2}\right) u$, where $k$ is a real scalar are examples of the elliptic operator $L$. The Cauchy problem (1.1) is ill-posed in the 
sense that small pertubations of the boundary data $f$ and $g$ produces large errors in the solution, see [19].

Let us consider the following example to illustrate the concept of ill-posedness. Consider the following Cauchy problem for the 2-dimensional Laplace equation.

$$
\begin{cases}\Delta u_{n}(x, y)=0 & (x, y) \in(0,1) \times(0,1), \\ u_{n}(x, 0)=0 & x \in(0,1), \\ \partial_{y} u_{n}(x, 0)=\phi_{n}(x) & x \in(0,1) \\ u_{n}(0, y)=u_{n}(1, y)=0 & y \in(0,1)\end{cases}
$$

Let $\phi_{n}(x)=\frac{\sin n \pi x}{n \pi}$ with $n$ a positive integer. Using the method of separation of variables, the solution to (1.2) is given by

$$
u_{n}(x, y)=\frac{\sin n \pi x \sinh n \pi y}{(n \pi)^{2}}
$$

The sequence $\phi_{n}$ tends to zero as $\mathrm{n}$ tends to infinity while for a fixed $y>0$, the solution $u_{n}(x, y)$ of $(1.2)$ tends to infinity. Therefore the requirement that the solution should depend continuously on the given data is not fulfilled and the problem is ill-posed.

Failure of the solution to depend continuously on the Cauchy data is the main challenge in the numerical solution of Cauchy problems for elliptic equations. Classical numerical methods cannot stably approximate solutions to such problems. Regularization methods are applied instead. Regularization methods involves reformulation of the problem so that solution to the regularized problem is less sensitive to pertubations. A number of regularization methods have been proposed for solving Cauchy problems for elliptic equations. These include Tikhonov-type regularization methods and iterative type regularization methods. See [20, 19, 4, 17, 8] for more details on ill-posed problems and regularization methods.

Our motivation for using an iterative method is that apart from the fact that it is easy to implement, it can also be applied to general geometries and to elliptic equations with constant coefficients as well as elliptic equations with variable coefficients.

We also note that, discrete approximation of solutions to Cauchy problems for elliptic equations in unbounded domains are computed on bounded domains which are obtained from unbounded domains by an appropriate truncation.

In the next section we present the iterative method proposed by Kozlov and Maz'ya[13] for ill-posed elliptic problems in bounded domains, which forms the basis of our work in this thesis.

\subsection{Alternating iterative procedure}

The alternating iterative procedure is an iterative regularization method introduced by Kozlov and Maz'ya [13] for solving ill-posed partial differential equations in bounded domains. This method involve alternatively solving a sequence of 
well-posed mixed boundary value problems for the same equation. The regularizing character is achieved by appropriate choice of boundary conditions at each iteration.

In [14], Kozlov et.al demonstrated that the alternating iterative procedure converges for Cauchy problems associated to linear, elliptic and positive-definite operators. They considered the Cauchy problem for the Laplace equation and Lame' system on a bounded domain $\Omega$ with a smooth boundary $\Gamma$ divided into two disjoint boundaries $\Gamma_{0}$ and $\Gamma_{1}$ with smooth common boundary. We use this example to illustrate how the alternating iterative procedure works.

Let $u$ be the exact solution to the following Cauchy problem for the Laplace equation:

$$
\Delta u=0 \quad \text { in } \quad \Omega, \quad u=f \quad \text { on } \quad \Gamma_{0}, \quad \partial_{\nu} u=g \text { on } \Gamma_{0}
$$

where $f, g$ are the specified Cauchy data.

The alternating iterative procedure for solving (1.3) consists of the following steps:

(1) Specify the initial approximation $\psi^{(0)}$ of the normal derivative on $\Gamma_{1}$ and solve the following well-posed mixed boundary problem to obtain the first approximation $u^{(0)}$

$$
\left\{\begin{array}{lll}
\Delta u^{(0)}=0 & \text { in } & \Omega \\
u^{(0)}=f & \text { on } & \Gamma_{0} \\
\partial_{\nu} u^{(0)}=\psi^{(0)} & \text { on } & \Gamma_{1}
\end{array}\right.
$$

(2) Having constructed the approximation $u^{(2 n)}$, the following well-posed mixed boundary value problem is solved to obtain the approximation $u^{(2 n+1)}$

$$
\left\{\begin{array}{lll}
\Delta u^{(2 n+1)}=0 & \text { in } & \Omega \\
\partial_{\nu} u^{(2 n+1)}=g & \text { on } & \Gamma_{0} \\
u^{(2 n+1)}=u^{(2 n)} & \text { on } & \Gamma_{1}
\end{array}\right.
$$

(3) Having constructed the approximation $u^{(2 n+1)}$, the following well-posed mixed boundary value problem problem is solved to obtain approximation $u^{(2 n+2)}$

$$
\begin{cases}\Delta u^{(2 n+2)}=0 & \text { in } \Omega \\ u^{(2 n+2)}=f & \text { on } \Gamma_{0} \\ \partial_{\nu} u^{(2 n+2)}=\partial_{\nu} u^{(2 n+1)} & \text { on } \Gamma_{1}\end{cases}
$$

The above mixed boundary value problems are well-posed and solvable in $H^{1}(\Omega)$ for appropriate function spaces for the Cauchy data $(f, g)$ on $\Gamma_{0}$ and the approximate normal derivative $\psi$ on $\Gamma_{1}$.

This original alternating iterative procedure introduced by Kozlov and Maz'ya includes iterations between Dirichlet-Neumann and Neumann-Dirichlet of the mixed boundary value problems. They used Dirichlet-Neumann mixed boundary conditions in their algorithm. It however does not necessarily converge if the elliptic 
operator is not positive-definite. Helmholtz-type operators are example of such operators.

Consider the following Cauchy problem for the Helmholtz equation.

$$
\left(\Delta+k^{2}\right) u=0 \text { in } \Omega \quad u=f \text { on } \Gamma_{0}, \quad \partial_{\nu} u=g \text { on } \Gamma_{0}
$$

where $k^{2}$ is the wave number. The problem is to reconstruct the solution to the Helmholtz equation from Cauchy data $(f, g)$ given on $\Gamma_{0}$.

It has been shown by Marin et.al [16] that in the case of the modified Helmholtz equation, that is when $k$ is purely imaginary, the Kozlov-Maz'ya alternating iterative procedure [14] always converges. However, if $k$ is real, the Kozlov-Maz'ya alternating iterative procedure does not converge for large values of $k^{2}$ in the Helmholtz equation, see [2].

In order to solve this problem of non-convergence for large values of $k^{2}$ in the Helmholtz equation, several variants of the Kozlov-Maz'ya alternating iterative procedure have been considered. See for examples Mpinganzima et.al $[2,3]$ and Johansson et.al [11] modifications. Johansson et.al [11], presented a modification of the Kozlov-Maz'ya alternating iterative procedure for Cauchy problems associated with elliptic operators which are symmetric but not positive and proved convergence of the modified algorithm. However, this algorithm by Johansson et.al is not easy to implement numerically.

Mpinganzima et.al [2] also presented a modification where they introduced an artificial interior boundary in such a way that convergence was restored. They also presented in [3], a simpler modification of the Kozlov-Maz'ya alternating iterative procedure for solving the Cauchy problem for the Helmholtz equation which is convergent even for large values of $k^{2}$ in the Helmholtz equation and easier to implement numerically.

The iterations consists of replacing the Dirichlet-Neumann iterations on $\Gamma_{1}$ by the Dirichlet-Robin iterations in the sequence solution of the following mixed boundary value problems:

$$
\begin{cases}\Delta u+k^{2} u=0 & \text { in } \quad \Omega \\ u=f & \text { on } \Gamma_{0} \\ \partial_{\nu} u+\mu u=\eta & \text { in } \Gamma_{1}\end{cases}
$$

and

$$
\left\{\begin{array}{lll}
\Delta u+k^{2} u=0 & \text { in } & \Omega \\
\partial_{\nu} u=g & \text { in } & \Gamma_{0} \\
u=\phi & \text { in } & \Gamma_{1}
\end{array}\right.
$$

For $(f, g) \in H^{\frac{1}{2}}\left(\Gamma_{0}\right) \times H^{-1 / 2}\left(\Gamma_{0}\right)$ if $\eta \in H^{-1 / 2}\left(\Gamma_{1}\right)$ and $\phi \in H^{\frac{1}{2}}\left(\Gamma_{1}\right)$ then the problems (1.8) and (1.9) are well-posed. Moreover, for appropriate choice of $\mu$ such that the quadratic form associated with the Helmholtz equation with Robin boundary conditions is positive, the Robin-Dirichlet alternating iterative procedure convergences in $H^{1}(\Omega)$ to the solution $u$ of the Cauchy problem (1.7) for any initial approximation $\eta \in H^{-1 / 2}\left(\Gamma_{1}\right)$. 
This thesis is based on this alternating iterative procedure proposed by Mpinganzima et.al [3]. In the next section we give a summary of the two papers in the thesis. 


\section{2 - Summary of Papers}

\section{Paper I}

In this paper, we analyse the Robin-Dirichlet alternating iterative procedure for Cauchy problem for general elliptic equations of second order. We consider the following Cauchy problem for an elliptic equation

$$
\begin{cases}L u=D_{j} a^{j i}(x) D_{i} u+a(x) u=0 & \text { in } \Omega, \\ u=f & \text { on } \Gamma_{0}, \\ N u=g & \text { on } \Gamma_{0},\end{cases}
$$

Here $\Omega$ is a bounded domain in $R^{d}$ with a Lipschitz boundary $\Gamma$ divided into two disjoint parts $\Gamma_{0}$ and $\Gamma_{1}$ with a common Lipschitz boundary in $\Gamma . D_{j}=\partial / \partial x_{j}$, $a^{j i}$ and $a$ are measurable real valued functions such that $a$ is bounded, $a^{i j}=a^{j i}$ and

$$
\lambda|\xi|^{2} \leq a^{i j}(x) \xi_{i} \xi_{j} \leq \lambda^{-1}|\xi|^{2}, \quad x \in \Omega, \quad \xi \in R^{d}, \quad \lambda=\text { const }>0
$$

The conormal derivative $N$ is defined as

$$
N u=\nu a^{i j} D_{i} u
$$

where $\nu=\left(\nu_{1}, \cdots, \nu_{d}\right)$. The Cauchy data $(f, g) \in H^{\frac{1}{2}}\left(\Gamma_{0}\right) \times H^{-1 / 2}\left(\Gamma_{0}\right)$.

We make one of the following two equivalent assumptions.

$$
\int_{\Omega}\left(a^{j i} D_{i} u D_{j} u-a u^{2}\right) d x>0 \text { for all } u \in H^{1}(\Omega, \Gamma) \backslash\{0\}
$$

where $H^{1}(\Omega, \Gamma)$ is the space of all functions from $H^{1}(\Omega)$ which vanish on $\Gamma$ and for two real valued measurable bounded functions $\mu_{0}$ and $\mu_{1}$ defined on $\Gamma_{0}$ and $\Gamma_{1}$ respectively,

$$
\int_{\Omega}\left(a^{j i} D_{i} u D_{j} u-a u^{2}\right) d x+\int_{\Gamma_{0}} \mu_{0} u^{2} d S+\int_{\Gamma_{1}} \mu_{1} u^{2} d S>0
$$

for all $u \in H^{1}(\Omega) \backslash\{0\}$. We note that in (2.2), we require that functions are equal to zero on the boundary while (2.3), we do not require that.

With the assumptions (2.3) in place, the Robin-Dirichlet alternating iterative procedure is used to solve problem (2.1). See Section 2 for a complete description of the iterative procedure. Well-posedness of the mixed boundary value problems used in the Robin-Dirichlet alternating iterative procedure are proved in the space $H^{1}(\Omega)$, see Preposition 3.7. Convergence of the Robin-Dirichlet alternating iterative procedure to the solution of $(2.1)$ is proved in Theorem 4.1. Numerical experiments are conducted using the Cauchy problem for the Helmholtz equation. In the experiments, the precise behaviour of the Robin-Dirichlet 
alternating iterative procedure for different values of $k^{2}$ in the Helmholtz equation, is investigated. Also investigated is how the choice of the Robin parameters influence the convergence of the iterations.

\section{Paper II}

In this paper, we consider the case where $L$ in (2.1) is the Helmholtz operator and the problem is prescribed in an unbounded domain described as follows. Let $\Omega$ be a domain in $R^{d}, d \geq 2$, with $C^{2}$ boundary and with $N$ cylindrical outlets to infinity, i.e. for sufficiently large $|x|$ the domain $\Omega$ coincides with the union of $N$ disjoint cylinders $\mathcal{C}^{(j)}, j=1, \ldots, N$, which can be described in a certain cartesian coordinates $x^{(j)}=\left(y^{(j)}, z^{(j)}\right)$, as

$$
\mathcal{C}^{(j)}=\left\{x^{(j)}: y^{(j)} \in \omega^{(j)}, z^{(j)} \in R\right\},
$$

where the cross-sections $\omega^{(j)}$ are bounded domains in $R^{d-1}$ with $C^{2}$ boundaries. We denote the boundary of $\Omega$ by $\Gamma$. We assume that a certain bounded ${ }^{1}$ open set $\Gamma_{0}$ is chosen on the boundary $\Gamma$ and the boundary of this set is of class $C^{2}$ also. Let also $\Gamma_{1}$ be the interior of $\Gamma \backslash \Gamma_{0}$.

We then seek a real valued solution $u \in H^{1}(\Omega)$ to the following Cauchy problem for the Helmholtz equation

$$
\left(\Delta+k^{2}\right) u=0 \text { in } \Omega
$$

and

$$
u=f_{0} \quad \text { on } \quad \Gamma_{0}, \quad \partial_{\nu} u=g_{0} \quad \text { on } \quad \Gamma_{0}
$$

where $k$ is a non-negative number, $\nu$ is the outward unit normal to $\Gamma, \partial_{\nu}$ is the normal derivative. The Cauchy data $\left(f_{0}, g_{0}\right) \in H^{\frac{1}{2}}\left(\Gamma_{0}\right) \times H^{-1 / 2}\left(\Gamma_{0}\right)$.

One of the following two equivalent assumption concerning the parameter $k$ are made. There exist a positive constant $\epsilon$ such that

$$
\int_{\Omega}\left(|\nabla u|^{2}-k^{2}|u|^{2}\right) d x \geq\left.\epsilon|| u\right|_{H^{1}(\Omega)} ^{2} \text { for all } u \in H^{1}(\Omega, \Gamma) .
$$

and there exist positive constants $\mu_{0}, \mu_{1}$ and $\delta$ such that

$$
\int_{\Omega}\left(|\nabla u|^{2}-k^{2}|u|^{2}\right) d x+\mu_{0} \int_{\Gamma_{0}}|u|^{2} d S+\mu_{1} \int_{\Gamma_{1}}|u|^{2} d S \geq \delta \|\left. u\right|_{H^{1}(\Omega)} ^{2}
$$

for all $u \in H^{1}(\Omega)$.

As in Paper 1, the Robin-Dirichlet alternating iterative procedure is proposed to solve problem (2.4) and (2.5). Complete description of the procedure is presented in section 1.1. Condition (2.6) is analysed and explicit estimates for $k^{2}$ in terms of eigenvalues of certain auxiliary problems are presented, see Lemma 2.2. Equivalence of condition (2.6) and (2.7) are also proved, see Lemma 2.3. The relationship between the first eigenvalue of the Robin-Laplacian and the first eigenvalue of the Dirichlet-Laplacian are established, see Example 2.5. An

\footnotetext{
${ }^{1}$ This is a set where measurements are taken and it is reasonable to assume it bounded
} 
example illustrating how to explicitly calculate the first eigenvalue of the Robin-Laplacian in a domain $\Omega$ in $R^{2}$ is presented in Example 2.4. Also included is a table showing linear dependence of the first eigenvalue of the Robin-Laplacian on the Robin parameters $\mu_{0}$ and $\mu_{1}$, see Table 1 . In the numerical experiments we demonstrate that by appropriate truncation of the domain and with appropriate choice of the Robin parameters $\mu_{0}$ and $\mu_{1}$, the Robin-Dirichlet alternating iterative procedure is convergent.

\section{References}

[1] Alvin Bayliss, Max Gunzburger, and Eli Turkel. Boundary conditions for the numerical solution of elliptic equations in exterior regions. SIAM Journal on Applied Mathematics, 42(2):430-451, 1982.

[2] Fredrik Berntsson, VA Kozlov, Lydie Mpinganzima, and Bengt-Ove Turesson. An alternating iterative procedure for the Cauchy problem for the Helmholtz equation. Inverse Problems in Science and Engineering, 22(1):45-62, 2014.

[3] Fredrik Berntsson, Vladimir Kozlov, Lydie Mpinganzima, and Bengt Ove Turesson. RobinDirichlet algorithms for the Cauchy problem for the Helmholtz equation. Inverse Problems in Science and Engineering, 26(7):1062-1078, 2018.

[4] A. L. Bukhgeim, J. Cheng, and M. Yamamoto. Uniqueness and stability for an inverse problem of determining a part of boundary. In Inverse problems in engineering mechanics (Nagano, 1998), pages 327-336. Elsevier, Oxford, 1998.

[5] D. Colton and R. Kress. Inverse Acoustic and Electromagnetic Scattering Theory. Springer-Verlag, 2nd edition, 1998.

[6] T. Delillo, V. Isakov, N. Valdivia, and L. Wang. The detection of the source of acoustical noise in two dimensions. SIAM J. Appl. Math., 61(6):2104-2121, 2001.

[7] T. Delillo, V. Isakov, N. Valdivia, and L. Wang. The detection of surface vibrations from interior acoustical pressure. Inverse Problems, 19:507-524, 2003.

[8] H. Engl, M. Hanke, and A. Neubauer. Regularization of Inverse Problems. Kluwer Academic Publishers, Dordrecht, the Netherlands, 1996.

[9] J. Hadamard. Lectures on Cauchy's problem in linear partial differential equations. Dover Publications, New York, 1953.

[10] Jacques Hadamard. Sur les problèmes aux dérivées partielles et leur signification physique. Princeton university bulletin, pages 49-52, 1902. 
[11] B.T Johansson and V.A Kozlov. An alternating method for Helmholtz-type operators in non-homogeneous medium. IMA Journal of Applied Mathematics, 74:62-73, 2009.

[12] D.S. Jones. Acoustic and Electromagnetic Waves. Clarendon Press, 1986.

[13] V.A. Kozlov and V.G. Maz'ya. Iterative procedures for solving ill-posed boundary value problems that preserve the differential equations. Algebra $i$ Analiz, 1(5):144-170, 1989. translation in Leningrad Math. J. 1(1990), no. 5, pp. 1207-1228.

[14] V.A. Kozlov, V.G. Maz'ya, and A.V. Fomin. An iterative method for solving the cauchy problem for elliptic equations. Comput. Maths. Math. Phys., 31(1):46-52, 1991.

[15] C. Langrenne and A. Garcia. Data completion method for the characterization of sound sources. J. Acoust. Soc. Am., 130(4):2016-2023, 2011.

[16] L Marin, L Elliott, PJ Heggs, DB Ingham, D Lesnic, and X Wen. An alternating iterative algorithm for the Cauchy problem associated to the Helmholtz equation. Computer methods in applied mechanics and engineering, 192(56):709-722, 2003.

[17] David L Phillips. A technique for the numerical solution of certain integral equations of the first kind. Journal of the ACM (JACM), 9(1):84-97, 1962.

[18] A. Schuhmacher, J. Hald, H.B. Rasmussen, and P.C. Hansen. Sound source reconstruction using inverse boundary element calculations. J. Acoust. Soc. Am., 113(1):114-127, 2003.

[19] Andrei N Tikhonov. Solution of incorrectly formaulated problems and the regularization method. In Dokl. Akad. Nauk., volume 151, pages 1035-1038, 1963.

[20] Andrei Nikolaevich Tikhonov. Regularization of incorrectly posed problems. Soviet Mathematics Doklady, 1963. 


\section{Papers}

The papers associated with this thesis have been removed for copyright reasons. For more details about these see:

http://urn.kb.se/resolve?urn=urn:nbn:se:liu:diva-170835 


\section{FACULTY OF SCIENCE AND ENGINEERING}

Linköping Studies in Science and Technology

Licentiate Thesis No. 1891, 2020

Department of Mathematics [MAI]

Linköping University

SE-581 83 Linköping, Sweden

www.liu.se 